\title{
Dinâmica Demográfica dos Kamaiurá, povo Tupi do Parque Indígena do Xingu, Mato Grosso, Brasil, 1970-1999*
}

\author{
Heloisa Pagliaro** \\ Carmen Junqueira*** \\ Luciana Garrido dos Santos Mendaña*** \\ Sofia Beatriz Mendonça***** \\ Roberto Geraldo Baruzzi ${ }^{\star \star \star \star \star \star}$
}

\begin{abstract}
Este trabalho analisa a dinâmica demográfica dos Kamaiurá, povo do tronco lingüístico Tupi, que, juntamente com outros nove povos das línguas Aruak, Karib, Tupi e Trumai, habitam o Parque Indígena do Xingu (PIX), ao norte do Mato Grosso. As fontes de dados são as fichas médicas e os livros de registro de eventos vitais do arquivo do Programa de Saúde da Universidade Federal de São Paulo no PIX, alimentado regularmente desde 1965. As medidas demográficas estimadas são: composição por idade e sexo; taxas brutas de natalidade; taxas de fecundidade total; taxas de mortalidade geral e taxas de mortalidade específicas por idade e sexo; mortalidade proporcional por sexo e idade; e taxas de mortalidade infantil. $O$ estudo mostra que os Kamaiurá estão vivenciando um processo de recuperação populacional, com crescimento de 3,1\% ao ano, entre 1970 e 1999. Altos níveis de fecundidade contribuíram para a expansão das novas gerações Kamaiurá, garantindo a sobrevivência física do grupo. Níveis de mortalidade relativamente baixos e estáveis resultaram da melhoria das condições de saúde, o que propicia aumento da sobrevivência de crianças, jovens, adultos e idosos, assegurando maior longevidade à população e manutenção de sua organização social.
\end{abstract}

Palavras-chave: Kamaiurá. Demografia dos povos indígenas. Índios da América do Sul. Alto Xingu.

\section{Introdução}

Os Kamaiurá são um povo de língua Tupi que, juntamente com outras nove tribos de línguas Aruak, Karib, Tupi e Trumai, habitam a região dos formadores do Rio Xingu, ao norte do estado do Mato Grosso. A grande homogeneidade cultural entre essas tribos é evidenciada em múltiplos aspectos, como a forma e a disposição das aldeias, tipo de habitação, hábitos alimentares, reclusão pubertária, pinturas e adornos corporais, uso do uluri ${ }^{1}$ pelas mulheres, festas e cerimônias, como o Kuarup de caráter intertribal. Este padrão cultural comum teria resultado da longa ocupação de uma

\footnotetext{
* Versao preliminar deste trabalho foi apresentada no XIV Encontro Nacional de Estudos Populacionais, Abep, realizado em Caxambu - MG - Brasil, de 20 a 24 de setembro de 2004.

** Demógrafa, Unifesp/EPM, doutora em Saúde Pública.

${ }^{* * *}$ Antropóloga, professora titular da Universidade Católica de São Paulo, pós-doutora em Antropologia.

**** Graduanda em Ciências Médicas, Unifesp/EPM; bolsista Pibic.

$* * * \star *$ Médica, Projeto Xingu/Unifesp/EPM, mestre em Antropologia.

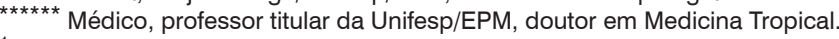

1 Pequeno cinto pubiano.
} 
mesma área geográfica e da freqüência de casamentos intertribais.

As primeiras referências aos Kamaiurá foram feitas em 1884 e 1887, por Karl von den Steinen (1940; 1942), que encontrou estes índios próximos da lagoa do Ipavu, no Alto Xingu. Após Steinen, no final do século XIX e início do XX, cientistas estrangeiros e brasileiros estiveram no Alto Xingu, permitindo um melhor conhecimento da região e de seus habitantes.

Quando o antropólogo Kalervo Öberg visitou o Alto Xingu, em 1948, os Kamaiurá haviam abandonado sua aldeia nas proximidades da Lagoa do Ipavu, em razão de uma epidemia de gripe, e estavam morando às margens do Rio Tuatuari, afluente da margem direita do Rio Culuene, numa aldeia que tinha seis casas e 110 habitantes (ÖBERG, 1953). Também em 1948, Eduardo Galvão, antropólogo do Museu Nacional, esteve na aldeia do Tuatuari, onde realizou estudo sistemático sobre os Kamaiurá. A aldeia então possuía cinco casas, pois uma havia sido incendiada e estava sendo reconstruída, e a população era de 55 homens e 55 mulheres (Galvão, 1979).

Em 1954, uma epidemia de sarampo, que teve início no Jacaré, antigo Posto da Fundação Brasil Central, no Rio Culuene, próximo ao Posto Indígena Capitão Vasconcelos, acometeu uma população de cerca de 600 índios do Alto Xingu, causando 114 óbitos, entre os quais 15 da etnia Kamaiurá (MOTTA, L., 1954; MOTTA, S., 1954).

A recuperação da história recente dos Kamaiurá confirma que, antes de 1970, a mortalidade era alta devido às disputas entre os povos da região e às epidemias de doenças contagiosas introduzidas pelo contato com não-índios. A fecundidade era moderada, graças a um padrão tradicional de família de dois a três filhos, obtido pelo emprego de práticas culturais de controle da natalidade, como uso de ervas contraceptivas, práticas de aborto, tabus pós-parto e aleitamento prolongado (JUNQUEIRA, 1978). Os altos níveis de mortalidade e a prática de infanticídio também teriam contribuído para manter esse tamanho de família e um volume populacional estável.
O presente estudo visa analisar a dinâmica demográfica dos Kamaiurá, no período 1970-1999, de forma a contribuir para a avaliação da atenção à saúde oferecida a esse povo desde 1965 e ampliar o conhecimento demográfico e epidemiológico sobre os povos indígenas no Brasil.

\section{Material e métodos}

Este estudo baseia-se em informações secundárias referentes ao período de $1970 \mathrm{a}$ 1999. Os dados quantitativos básicos foram extraídos das fichas médicas e dos livros de registro de eventos vitais do arquivo do Programa de Saúde da Universidade Federal de São Paulo/Escola Paulista de Medicina (Unifesp/EPM) no Parque Indígena do Xingu (PIX), o qual é realimentado regularmente pelas equipes de saúde desde 1965, ano em que teve início o programa, que se estende até o presente.

As fichas médicas possuem, além das informações de saúde: número de registro no programa de saúde; fotos de identificação na infância, adolescência, idade adulta e velhice; etnia a que pertence; data de nascimento estimada pela equipe médica que realizou o primeiro exame clínico dos indivíduos nascidos antes da implantação do Programa e mês e ano para os nascidos a partir de 1966; os diferentes nomes que os indivíduos recebem durante os ciclos de vida; sexo; data de óbito, local e causa de morte; nome dos pais e cônjuge(s); localidade ou aldeia de residência e eventuais mudanças; nome e número de registro dos filhos.

Os livros de registro de eventos vitais possuem informações sobre nascimentos e óbitos ocorridos desde a implantação do sistema, tais como: número de registro no programa de saúde; nome; sexo; mês e ano de nascimento; etnia; data de abertura da ficha médica; número e nomes dos pais, quando conhecidos; mês e ano do óbito, se ocorreu; e causa de morte.

A alimentação contínua desse arquivo e a qualidade das suas informações permitiram identificar os eventos demográficos ocorridos entre os Kamaiurá de 1970 a 1999, bem como reconstruir a população por sexo 
e idade, para cada ano desse período, por meio do emprego de um Diagrama de Lexis, tomando-se como ponto inicial a população cadastrada até 1970, por idades simples e sexo, à qual foram sendo acrescentados, ano a ano, até 1999 , os nascimentos, os óbitos, as entradas e saídas das aldeias, nas idades em que estes eventos ocorreram.

O volume anual da população obtido por meio dessa técnica, nos anos de 1970 a 1999, serviu de base para estimar as seguintes medidas demográficas: composição por idade e sexo; taxas brutas de natalidade (TBN); taxas de fecundidade total (TFT); taxas brutas de mortalidade (TBM); taxas de mortalidade infantil (TMI); mortalidade proporcional; e taxas específicas de mortalidade por sexo e idade. Para contornar as flutuações dos indicadores estimados, resultantes do pequeno volume populacional, calcularam-se indicadores médios para períodos de dez anos.

\section{Dinâmica da população}

Em 1970, a aldeia Kamaiurá possuía sete casas e uma população de 136 habitantes. Uma cisão do grupo levou à abertura de uma segunda aldeia no Morená, em 1985, às margens do Rio Xingu, local sagrado dos Kamaiurá, onde viveu Mavutsnin, criador dos índios do Alto Xingu. Em 1999, as aldeias do Ipavu e Morená possuíam, respectivamente, 18 e cinco casas, com 341 habitantes.

O crescimento da população Kamaiurá, de $2,6 \%$ ao ano no período $1970-79$, estabilizou-se num patamar que variou entre 3,6\% e $3,2 \%$ nos anos subseqüentes, resultando numa taxa média de $3,1 \%$ ao ano no período 1970-99 (Tabelas 1 e 2).

$\mathrm{O}$ crescente número de nascimentos e o reduzido número de mortes contribuíram para que a população alcançasse este ritmo de crescimento, tendo sido irrelevantes as entradas e saídas da população. Entre 1970 e 1999, ocorreram 273 nascimentos e 50 mortes, acarretando um incremento vegetativo de 223 indivíduos. Nesse período, o saldo migratório resultante de casamentos intertribais foi negativo (-12). O resultado desse crescimento intrínseco e extrínseco foi o aumento líquido de 205 indivíduos, equivalendo a um volume populacional 1,5 vez superior ao da população inicial, de 136 pessoas em 1970.

Dados da história recente dos Kamaiurá apontam para a estabilidade de seu volume populacional, em torno de 110 habitantes,

TABELA 1

População Kamaiurá segundo sexo, razões de sexo e distribuição por grupos etários Parque Indígena do Xingu, MT, Brasil - 1970-1999

\begin{tabular}{lcccc}
\hline Indicadores & $\mathbf{1 9 7 0}$ & $\mathbf{1 9 7 9}$ & $\mathbf{1 9 8 9}$ & $\mathbf{1 9 9 9}$ \\
\hline População & $\mathbf{1 3 6}$ & $\mathbf{1 7 5}$ & $\mathbf{2 5 0}$ & $\mathbf{3 4 1}$ \\
Homens & 75 & 94 & 124 & 169 \\
Mulheres & 61 & 81 & 126 & 172 \\
& & & $\mathbf{9 8 , 3}$ \\
Razões de sexo (por cem) & $\mathbf{1 2 3}$ & $\mathbf{1 1 6 , 1}$ & $\mathbf{9 8 , 4}$ & $\mathbf{1 1 1 , 5}$ \\
Menos de 15 anos & 84,9 & 92,5 & 93,6 & 80,2 \\
15 a 49 anos & 165,4 & 125,6 & 100,0 & 116,7 \\
50 anos e mais & 200,0 & 400,0 & 115,4 & $\mathbf{1 0 0 , 0}$ \\
& & & $\mathbf{1 0 0 , 0}$ & 47,7 \\
Grupos etários (\%) & 100,0 & $\mathbf{1 0 0 , 0}$ & 48,3 & 41,0 \\
15 a 49 anos & 44,9 & 45,6 & 41,0 & 11,3 \\
\hline 0 anos e mais & 50,7 & 48,9 & 10,7 & \\
\hline
\end{tabular}

Fonte dos dados brutos: Projeto Xingu/DMP/Unifesp/EPM. 
no período de quase duas décadas que se estendeu entre 1948 e 1966 (OBERG, 1953; GALVÃO, 1979; JUNQUEIRA, 1978). A alta mortalidade, principalmente aquela registrada por ocasião de uma epidemia de sarampo que acometeu os povos do Alto Xingu em 1954, foi responsável pela manutenção dessa estabilidade (MOTTA, L. 1954; MOTTA, S. 1954).

Ritmo de crescimento semelhante ao da população Kamaiurá, no período de 1970-99 ( $3,1 \%$ ao ano), vem sendo observado, nas últimas décadas, em diversas populações indígenas no Brasil e na América Latina (MCSWEENEY; ARPS, 2005). Entre os Suyá, também moradores do PIX, verificou-se um crescimento médio anual, entre 1970 e 2004, de 3\% (PAGLIARO et al., 2007). Já entre os Juruna e os Kaiabi, habitantes da região central e norte do Parque Indígena do Xingu, o ritmo de crescimento médio anual verificado nesse mesmo período foi de 4,9\% e $5,2 \%$, respectivamente (PAGLIARO, 2005). Entre os Xavánte de Pimentel Barbosa e de Sangradouro-Volta Grande (MT), o incremento foi próximo de $5 \%$ ao ano (COIMBRA et al., 2002; SOUZA; SANTOS, 2001).

\section{Mortalidade}

As taxas brutas de mortalidade (TBM) observadas para o conjunto da população declinaram de 8,5 para 7,1 óbitos por mil habitantes, entre os períodos de 1970-79 e 1990-99 (Tabela 2). Considerando-se as TBMs padronizadas por meio de técnica direta, segundo o padrão etário da população Kamaiurá, o declínio da mortalidade foi ainda maior, passando de 17,2 óbitos por mil habitantes, em 1970-79, para 7,1, em 1990-99. Na população masculina foram registrados os mais altos níveis de mortalidade geral.

Esses resultados indicariam que a transformação do padrão demográfico dos Kamaiurá começou a se esboçar a partir dos anos 70 , quando teve início um processo de declínio da mortalidade, decorrente das medidas médico-sanitárias implementadas de maneira mais sistemática a partir de 1965, com a implantação de um programa de atenção à saúde dos povos do Parque Indígena do Xingu, coordenado pela Escola Paulista de Medicina, atual Universidade Federal de São Paulo (BARUZZI, 2005; RODRIGUES, 2005).

A despeito das dificuldades advindas do pequeno volume populacional e da subenumeração de óbitos em razão da prática de infanticídio, cultivada ainda hoje pelos Kamaiurá, as estimativas de níveis e padrões de mortalidade apontam para resultados que permitem comparar o seu comportamento ao de outros povos habitantes da região do Xingu. A sobremortalidade masculina registrada entre os Kamaiurá é uma tendência já observada em outros povos indígenas habitantes do PIX (PAGLIARO, 2005), com

TABELA 2

Taxas de fecundidade total (TFT), taxas brutas de natalidade (TBN), taxas brutas de mortalidade observadas e padronizadas (TBM, TBMp), taxas de crescimento vegetativo (TCV), taxas de crescimento médio anual (TCMA) e taxas de mortalidade infantil (TMI) da população Kamairá Parque Indígena do Xingu, MT, Brasil - 1970-1999

\begin{tabular}{lccc}
\hline Indicadores & $\mathbf{1 9 7 0 / 7 9}$ & $\mathbf{1 9 8 0 / 8 9}$ & $\mathbf{1 9 9 0 / 9 9}$ \\
\hline TFT (filhos nascidos vivos/mulher) & 5,4 & 6,6 & 6,4 \\
TBN (por mil) & 37,3 & 44,3 & 41,0 \\
TBM (por mil) & 8,5 & 7,5 & 7,1 \\
TBMp (por mil) & 17,2 & 10,8 & 7,1 \\
TCV (por mil) & 28,8 & 36,8 & 34,0 \\
TCMA (por cem) & 2,6 & 3,6 & 3,2 \\
TMI (por mil) & - & 21,3 & 32,8 \\
\hline
\end{tabular}

Fonte dos dados brutos: Projeto Xingu/DMP/Unifesp/EPM. 
exceção dos Suyá, sociedade em que há sobremortalidade feminina (PAGLIARO et al., 2007).

A Tabela 3 exibe a mortalidade proporcional por sexo e grupos etários, nos períodos de 1970-79, 1980-89 e 1990-99. Para o conjunto da população, as proporções de óbitos em menores de um ano de idade foram de $12,5 \%$ e $14,3 \%$, respectivamente, em 1980-89 e 1990-99, os dois períodos em que houve registro de mortes deste grupo etário. Estas proporções podem ser consideradas baixas se comparadas à dos Xavánte de Sangradouro-Volta Grande (MT), 55,2\%, no período 1993-97 (SOUZA; SANTOS, 2001).

Há alta probabilidade de sub-registro de óbitos de menores de um ano de idade entre os Kamaiurá, devido à prática cultural de infanticídio nessa população, a qual vem sendo combatida desde a chegada dos brancos na região do Xingu. Conforme Junqueira (1978 e 2002), o infanticídio é eticamente aceito entre os Kamaiurá como forma de impedir a sobrevivência de gêmeos, de crianças mal formadas fisicamente ou concebidas em situações em que o status da mulher leva ao abortamento provocado. Quando as tentativas de aborto fracassam - infusões de ervas e processos mecânicos-, os recém-nascidos são enterrados logo após o nascimento. As circunstâncias em que esta prática ocorre podem variar entre gravidez de viúvas em período de luto, quando devem se abster de relações sexuais, gravidez de mulheres solteiras cujo parceiro não deseja a união, situação em que a mãe não pode arcar com a responsabilidade de criar o filho, e mulheres casadas que engravidam em conseqüência de relações extraconjugais (JUNQUEIRA 1978 e 2002).

Em estudo sobre os Mucajai Yanomama, Early e Peters (1990) encontraram uma alta freqüência de abortos e infanticídios. Segundo os autores, os Yanomama consideram o infanticídio um aborto terminal e não um homicídio. A maioria das vezes em que apelam para o infanticídio, a rejeição do bebê, independentemente do sexo, é a causa mais freqüente, podendo ocorrer em razão do curto espaçamento entre os filhos, do nascimento de gêmeos, deformidades físicas, ausência de marido, separações e viuvez, e para selecionar o sexo dos filhos (SANTOS; PAGLIARO; AZEVEDO, 2005).

A proporção de óbitos registrados na população de 50 anos e mais cresceu de $7,7 \%$ para $28,6 \%$, entre as décadas de 70 e 90, acompanhando o incremento de população nessas idades, como pode ser observado mais adiante na análise da composição etária da população.

No estudo da mortalidade proporcional por sexo, chama a atenção a elevada pro-

TABELA 3

Distribuição dos óbitos da população Kamaiura, por sexo, segundo grupos etários Parque Indígena do Xingu, MT, Brasil - 1970-1999

Em porcentagem

\begin{tabular}{|c|c|c|c|c|c|c|c|c|c|}
\hline \multirow{2}{*}{ Grupos etários } & \multicolumn{3}{|c|}{$1970-79$} & \multicolumn{3}{|c|}{$1980-89$} & \multicolumn{3}{|c|}{$1990-99$} \\
\hline & Homens & Mulheres & Total & Homens & Mulheres & Total & Homens & Mulheres & Total \\
\hline Menos de 1 ano & - & - & - & 9,1 & 20,0 & 12,5 & 14,3 & 14,3 & 14,3 \\
\hline 1a 4 anos & 11,1 & 25,0 & 15,4 & 9,1 & 20,0 & 12,5 & 14,3 & _ & 9,5 \\
\hline 5 a 19 anos & 22,2 & _ & 15,4 & 27,3 & 20,0 & 25,0 & 21,4 & 28,6 & 23,8 \\
\hline 15 a 19 anos & 22,2 & _ & 15,4 & 27,3 & _ & 18,8 & 21,4 & 14,3 & 19,1 \\
\hline 20 a 49 anos & 66,7 & 50,0 & 61,5 & 27,3 & 40,0 & 31,2 & 28,6 & 14,3 & 23,8 \\
\hline 50 anos e mais & - & 25,0 & 7,7 & 27,3 & _ & 18,8 & 21,4 & 42,8 & 28,6 \\
\hline Total (\%) & 100,0 & 100,0 & 100,0 & 100,0 & 100,0 & 100,0 & 100,0 & 100,0 & 100,0 \\
\hline Total (abs.) & 9 & 4 & 13 & 11 & 5 & 16 & 14 & 7 & 21 \\
\hline
\end{tabular}

Fonte dos dados brutos: Projeto Xingu/DMP/Unifesp/EPM. 
porção de óbitos masculinos na faixa etária de 5 a 19 anos. A separação dos óbitos do grupo etário 15-19 anos do total da faixa 5-19 anos confirma esta afirmação. Ao longo do período estudado, o total de óbitos deste grupo etário concentrou-se nas idades de 15-19 anos, em proporções que correspondem, respectivamente, a $22,2 \%, 27,3 \%$ e $21,4 \%$ do total de óbitos masculinos, nas décadas de 70, 80 e 90. Para as mulheres, não foram registrados óbitos na faixa de 15 a 19 anos nas décadas de 70 e 80 , sendo de $14,3 \%$ a proporção de mortes de mulheres nestas idades no período 1990-99.

As taxas específicas de mortalidade por grupos etários e sexo dessa população - medida mais refinada de mortalidade, por ser menos influenciada pela estrutura etária - constam da Tabela 4. No conjunto da população, os mais elevados níveis de mortalidade são encontrados na faixa etária de 20 a 49 anos, na década de 70 , e na de menores de um ano, nas décadas de 80 e 90 . De um modo geral, a mortalidade masculina é superior à feminina em quase todos os grupos etários. No sexo masculino, destacam-se o grupo etário de 15 a 19 anos, na década de 80 (66,7 óbitos por mil), e de menores de um ano, no período 1990-99 (33,3 por mil). Para as mulheres, a mortalidade foi mais elevada nos grupos de 50 e mais anos, nas décadas de 70 (41,7 por mil) e de
90 (19,2 por mil), e entre os menores de um ano, na década de 80 (21,7 por mil).

Entre os Kamaiurá, existem tradicionalmente altos níveis de mortalidade na população masculina nas idades de 15 a 19 anos, em razão das praticas culturais de reclusão pubertária, rito de passagem realizado entre os povos do Alto Xingu e destinado ao desenvolvimento social, psicológico e físico do adolescente e seu ingresso no mundo adulto. Conforme Pinto e Baruzzi (2005), que estudaram o risco de morte associado à reclusão pubertária masculina, os óbitos decorreriam do uso de infusões ou da ação tópica de plantas nativas durante o rito de passagem. Os óbitos de jovens Kamaiurá registrados nessas idades, no período 197099, com exceção de um, foram causados por envenenamento, provocado pela ingestão de infusão de plantas nativas no ritual pubertário. Na seqüência destes óbitos, outras mortes ocorreram, desta feita por homicídios praticados contra os acusados de terem causado as mortes por feitiço dos jovens em reclusão.

Em razão desta prática cultural, a taxa de mortalidade dos homens nesse grupo etário, entre os Kamaiurá, atingiu 66 óbitos por mil habitantes, na década de 80 , sendo também expressiva nos demais períodos analisados. Para os Kaiabi, por exemplo, que não praticam esse rito, a taxa de mortalidade

TABELA 4

Taxas específicas de mortalidade da população Kamaiura, por sexo, segundo grupos etários Parque Indígena do Xingu, MT, Brasil - 1970-1999

Por mil habitantes

\begin{tabular}{|c|c|c|c|c|c|c|c|c|c|}
\hline \multirow{2}{*}{ Grupos etários } & \multicolumn{3}{|c|}{$1970-79$} & \multicolumn{3}{|c|}{$1980-89$} & \multicolumn{3}{|c|}{$1990-99$} \\
\hline & Homens & Mulheres & Total & Homens & Mulheres & Total & Homens & Mulheres & Total \\
\hline Menos de 1 & - & - & - & 23,3 & 20,4 & 21,7 & 33,3 & 16,9 & 25,2 \\
\hline 1 a 4 anos & 10,2 & 10,3 & 10,3 & 7,1 & 5,3 & 6,1 & 8,8 & _ & 4,5 \\
\hline 5 a 19 anos & 6,5 & _ & 3,4 & 8,4 & 2,7 & 5,5 & 5,3 & 3,2 & 4,2 \\
\hline 15 a 19 anos & 21,3 & - & 12,3 & 66,7 & - & 14,9 & 22,4 & 6,2 & 13,5 \\
\hline 20 a 49 anos & 17,4 & 7,6 & 13,2 & 7,5 & 6,1 & 8,2 & 9,5 & 2,2 & 5,7 \\
\hline 50 anos e mais & - & 41,7 & 12,2 & 25,4 & - & 9,8 & 15,3 & 19,2 & 17,0 \\
\hline Total & 10,7 & 5,8 & 8,5 & 10,3 & 4,9 & 7,7 & 9,5 & 4,7 & 7,1 \\
\hline
\end{tabular}

Fonte dos dados brutos: Projeto Xingu/DMP/Unifesp/EPM. 
na população masculina desta faixa etária foi de 3,2 óbitos por mil, entre 1970 e 1999 (PAGLIARO, 2005).

Cabe destacar que a análise da mortalidade infantil dos Kamaiurá foi prejudicada em razão do pequeno número de óbitos de menores de um ano declarados (apenas cinco), entre 1980 e 1999, devendo ser ressaltado que as crianças sacrificadas ao nascer em razão da prática cultural de infanticídio, na região do Alto Xingu, dificilmente são registradas pelo serviço médico da Unifesp (RODRIGUES et al., 2002).

\section{Natalidade e fecundidade}

As taxas brutas médias de natalidade (TBN) dessa população indicam tendência de aumento entre os períodos de 1970-79 e $1980-89$, de 37,3 para 44,3 nascimentos por mil habitantes, declinando na década de 90 para 41 nascimentos por mil habitantes (Tabela 2).

Os níveis de natalidade encontrados entre os Kamaiurá, que há séculos se localizam no Alto Xingu, seriam mais baixos do que os estimados para os Kaiabi, por exemplo, que começaram a migrar em direção ao Xingu em 1953, após conflitos com invasores de suas terras nos Rios Arinos e Teles Pires (MT), que causaram forte perda populacional. A partir de 1970, com a conquista da estabilidade territorial, eles teriam iniciado um processo de recuperação populacional. Para este povo, as taxas de natalidade variaram de 50 a 57 nascimentos por mil habitantes, também no período 1970-99 (PAGLIARO, 2005). Para os Xavánte de Pimentel Barbosa (MT), Flowers (1994) estimou uma taxa bruta média de natalidade de 51,4 nascimentos vivos por mil habitantes para o período 1977-90 e, para os Xavánte de Sangradouro-Volta Grande, a TBN estimada por Souza e Santos (2001) foi de 57,7 por mil, entre 1993 e 1997.

As taxas de fecundidade total (TFT) das mulheres Kamaiurá apresentam tendência de aumento entre as décadas de 70 e 80 , de 5,4 para 6,6 filhos nascidos vivos por mulher, em média, declinando ligeiramente na década de 90 para 6,4 filhos em média (Tabela 2).

Até os anos 60, os Kamaiurá possuíam um padrão de família relativamente pequeno, de dois a três filhos, em média (JUNQUEIRA, 1978; OBERG, 1953), cuja manutenção era garantida pela alta mortalidade, o conhecimento e a adoção de práticas de restrição voluntária dos nascimentos, como o uso de ervas de efeito contraceptivo, as práticas de aborto e de infanticídio, a existência de tabus pós-parto e o aleitamento prolongado (JUNQUEIRA, 1978; PAGLIARO; JUNQUEIRA, 2007). A partir da década de 70 , quando iniciaram-se o processo de declínio da mortalidade e o conseqüente aumento da sobrevivência de crianças e adultos, o tamanho de família dos Kamaiurá ampliou-se, embora se mantivessem, como o fazem até o presente, suas práticas tradicionais de contenção da natalidade. Nesta década, o número médio de filhos nascidos vivos por mulher já era de 5,7. Nos anos de 80 , quando o programa de saúde da Unifesp ampliou sua presença no Parque Indígena do Xingu, em razão da saída da Funai, a fecundidade dessas mulheres atingiu seu mais alto nível, 6,6 filhos em média por muIher, declinando ligeiramente para 6,4 filhos na década de 90

Apesar de esses valores parecerem bastante expressivos, o nível de fecundidade das mulheres Kamaiurá demonstrou ser inferior ao estimado para outros povos do PIX, como os Kaiabi, por exemplo, cuja TFT na década de 90 foi de 9,5 filhos nascidos vivos, em média, por mulher (PAGLIARO, 2005). A fecundidade das mulheres Kamaiurá seria também mais baixa do que a estimada para aquelas autodeclaradas indígenas no Censo Demográfico de 2000, residentes nas áreas rurais (6,9 filhos nascidos vivos por mulher) e no rural específico² (7,2 filhos) da região Centro-Oeste do Brasil (IBGE, 2005; WONG et al., 2006). Em relação ao conjunto da população brasileira, a fecundidade das

\footnotetext{
2 A denominação rural específico foi utilizada na análise das informações dos autodeclarados indígenas do Censo de 2000 para definir as áreas rurais dos municípios onde há terras indígenas.
} 
Kamaiurá encontra paralelo com os níveis registrados entre as décadas de 50 e 70 , quando as TFT oscilaram entre 5,8 e 6,3 filhos. Na atualidade, a TFT no Brasil é de 2,4 filhos em média, e na região Centro-Oeste do país, onde habitam os Kamaiurá, é de 2,3 (IBGE, 2004).

Conforme Pagliaro e Junqueira (2007) demonstraram em detalhado estudo sobre a fecundidade das Kamaiurá, os níveis de fecundidade dessas mulheres estão associados a padrões reprodutivos caracterizados por curtos intervalos de tempo entre os nascimentos, estimados entre 30 e 35 meses no período 1970-79, e ao aleitamento prolongado, que pode chegar até a três anos. Esta relação foi encontrada em outras sociedades indígenas no Brasil, como os Mekranoti (WERNER, 1983), os Mucajai Yanomama (EARLY; PETERS, 1990), os Xavánte de Pimentel Barbosa (FLOWERS, 1994; COIMBRA et al., 2002) e de Sangradouro-Volta Grande (SOUZA; SANTOS, 2001), os Kaiabi (PAGLIA-
RO, 2005) e alguns povos da região do Rio Negro (AZEVEDO, 2004).

\section{Estrutura da população}

A evolução da estrutura por idade e sexo da população Kamaiurá pode ser visualizada por meio das pirâmides de 1970 e 1999, apresentadas nos Gráficos 1 e 2.

A pirâmide de 1970 tem forma muito irregular e retrata as diversas gerações de Kamaiurá sobreviventes naquele ano, refletindo os altos níveis de mortalidade verificados nessa população nos anos 50 e em décadas anteriores, sobre os quais muito pouco se conhece. A forma bastante irregular desta pirâmide, principalmente no que tange à população masculina, deve-se ao pequeno volume da população e à sobremortalidade masculina, já avaliada anteriormente.

As bases das pirâmides de 1970 e 1999 permanecem semelhantes $(18,4 \%$ em $1970 \mathrm{e}$ $18,7 \%$ em 1999), com aumento da proporção

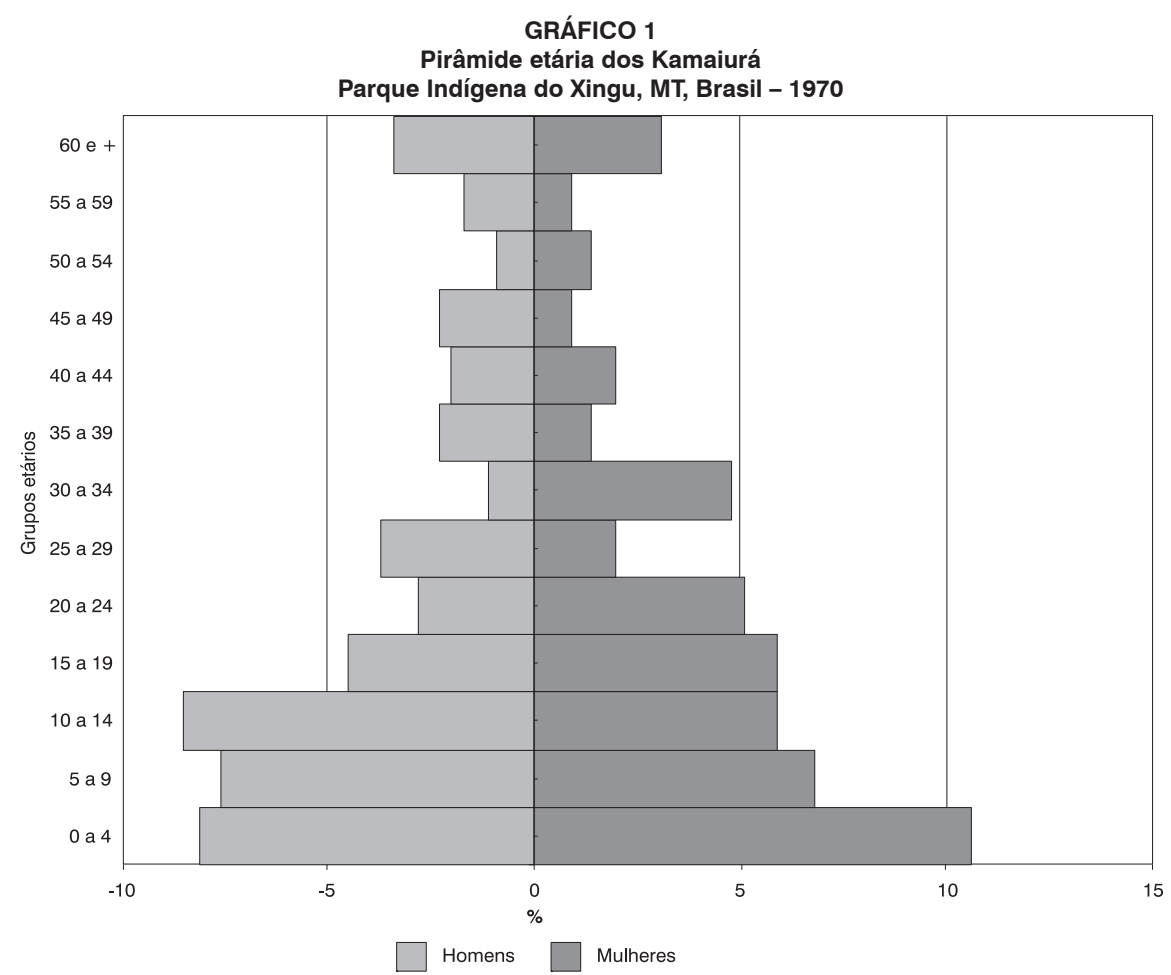

Fonte dos dados brutos: Projeto Xingu/DMP/Unifesp/EPM. 
de população do sexo masculino de 0 a 4 anos (de 5,9\%, em 1970, para 8,1\%, em 1999) e redução para o sexo feminino (de 12,5\% para $10,6 \%$ no mesmo período).

A pirâmide de 1999 possui contornos mais regulares que a de 1970 e desenho compatível com o de populações indígenas de perfil jovem, destacando-se o estreitamento nas idades adultas e o prolongamento e alargamento da cúspide que reflete o aumento do peso relativo da população com 50 ou mais anos.

O padrão etário caracterizado pela manutenção de uma estrutura jovem, com sinais de envelhecimento, confirma-se no exame da distribuição da população por grandes grupos etários (Tabela 1). A participação do segmento com menos de 15 anos de idade sobre o conjunto da população aumentou de $44,9 \%$ para $47,7 \%$, entre 1970 e 1999 , e a daqueles de 50 ou mais anos quase triplicou (de 4,4\% para 11,3\%), repercutindo na redução das proporções do grupo de 15 a 49 anos (de $50,7 \%$ para $41 \%$ ).
A evolução das razões de sexo da população, por grandes grupos etários, no período 1970-1999, pode ser observada na Tabela 1. Para o conjunto da população, esses dados estariam indicando a redução das RS, de 123 homens por cada cem mulheres, em 1970, para 98,3, em 1999. No grupo de menores de 15 anos, a predominância de mulheres na população persiste até 1989 , finalizando o período com 111,5 homens por cada cem mulheres, em 1999. Acompanhando a mudança de idades das gerações nascidas entre 1970 e 1985, na qual há predominância de mulheres, observa-se que, no grupo de 15 a 49 anos, a prevalência de população masculina diminui ao longo do tempo, sendo a RS de 80,2 homens por cem mulheres em 1999. Entre os maiores de 50 anos ainda há predominância de homens. Esses dados são consoantes com os encontrados por Pagliaro (2005) para os Kaiabi, também habitantes do PIX.

A evolução da composição por idade e sexo dessa população reflete a combinação

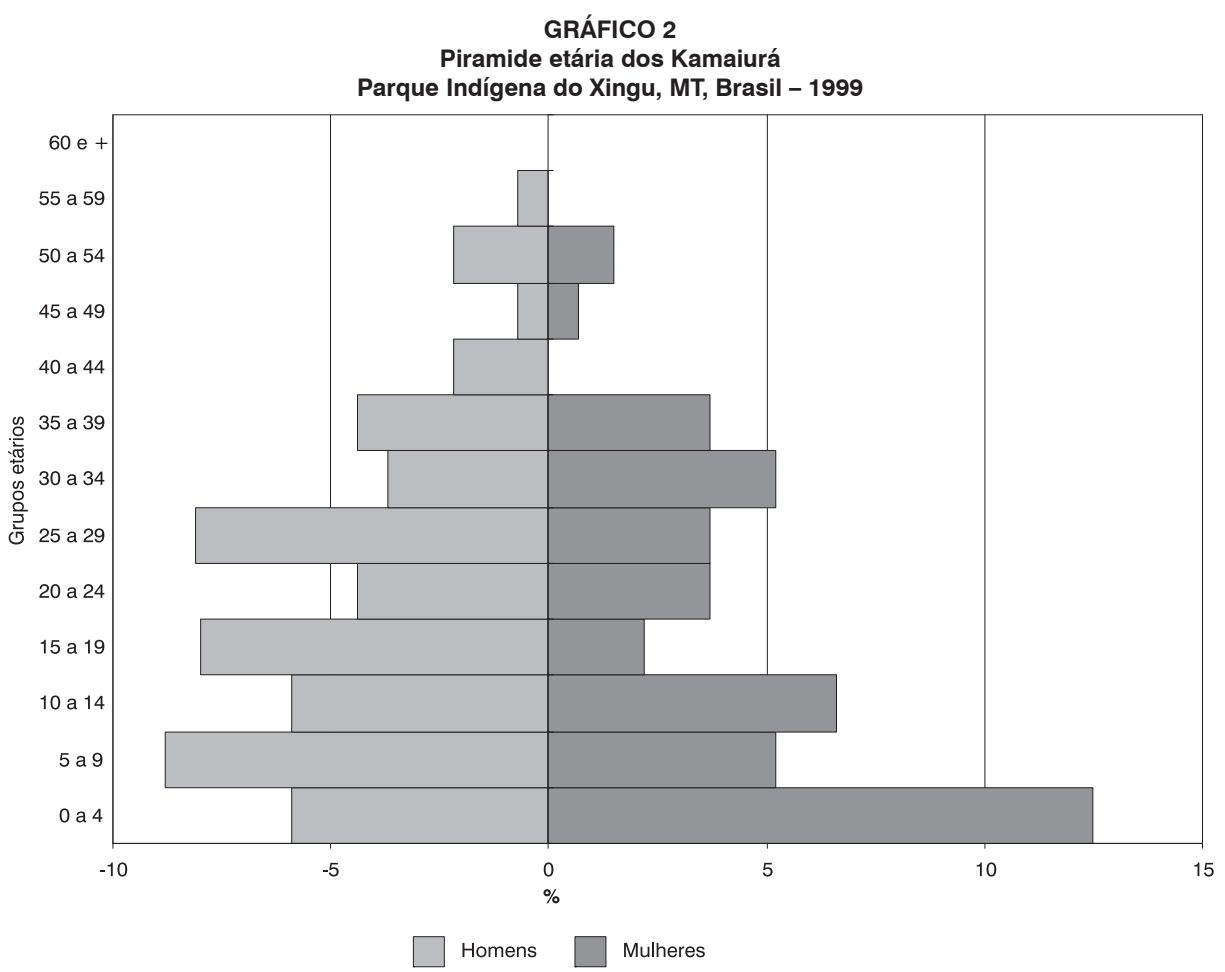

Fonte dos dados brutos: Projeto Xingu/DMP/Unifesp/EPM. 
entre declínio da mortalidade e aumento da fecundidade. A contribuição da queda da mortalidade para o aumento da sobrevivência dos filhos entre os Kamaiurá foi demonstrada por Pagliaro e Junqueira (2007), em estudo detalhado sobre a fecundidade desse povo. As autoras compararam a fecundidade acumulada das mulheres Kamaiurá, a partir da reconstrução de histórias reprodutivas do total de mulheres de 15 a 54 anos unidas e sobreviventes nos anos de 1971 e 2003, e demonstraram que o número médio de filhos sobreviventes cresceu de 2,4 para 4. Considerando os filhos nascidos vivos, exceto os sacrificados na prática cultural do infanticídio, a média de filhos dessas mulheres variou de 3,7 para 4,3 nesse período, tendo a média de filhos mortos (de todas as idades) diminuído de 1,3 para 0,3 por mulher. Ainda conforme as autoras, esse efeito da queda da mortalidade sobre o aumento da média de filhos por mulher seria decorrente das ações médicosanitárias implantadas, a partir de 1970 , pelo programa de saúde dos povos do Xingu, que teriam garantido a melhoria das condições de saúde da população e o aumento da sobrevivência de crianças e adultos (PAGLIARO; JUNQUEIRA, 2007).

\section{Considerações finais}

As modificações observadas no comportamento demográfico dos Kamaiurá

\section{Referências bibliográficas}

AZEVEDO, M. M. Demografia dos povos indígenas do Alto Rio Negro/Amazonas: um estudo de caso de nupcialidade e reprodução. Tese de Doutorado. Campinas, Unicamp, 2004.

BARUZZI, R. G. Do Araguaia ao Xingu. In: BARUZZI, R. G.; JUNQUEIRA, C. (Orgs.). Parque Indígena do Xingu. Saúde, cultura e história. São Paulo: Terra Virgem, 2005, p. 59-114.

COIMBRA JR., C. E. A.; FLOWERS, N. M.; SALZANO, F. M.; SANTOS, R. V. The Xavante in transition: health, ecology and bioanthropology in Central Brazil. Ann Arbor: University of Michigan Press; 2002. apontam para seu crescimento em ritmo bastante elevado, entre 1970 e 1999, acompanhando os níveis de crescimento encontrados entre outros grupos indígenas do PIX e de outras regiões do país (PAGLIARO; AZEVEDO; SANTOS, 2005).

A avaliação dos indicadores demográficos dessa população conduz à discussão sobre a relação existente entre padrões culturais e padrões demográficos dos povos indígenas, que, entre os Kamaiurá, configurase claramente na exposição de dois grupos etários a condições de alto risco de morte em razão de práticas culturais: o grupo de menores de um ano, sujeito ao infanticídio, cujas taxas de mortalidade estariam subestimadas; e o de rapazes de 15 a 19 anos, que estariam expostos à mortalidade por causas externas, como o envenenamento no período de reclusão pubertária.

Altos níveis de natalidade, apesar de inferiores àqueles observados nas últimas décadas do século XX entre algumas populações indígenas moradoras do território brasileiro, contribuíram para a expansão das novas gerações Kamaiurá, garantindo a sobrevivência física do grupo. Níveis de mortalidade relativamente baixos e estáveis resultaram da melhoria das condições de saúde, o que propiciou o aumento da sobrevivência de crianças, jovens, adultos e idosos, assegurando maior longevidade à população e manutenção de sua organização social.

EARLY, J. D.; PETERS, J. F. The population dynamics of the Mucajai Yanomama. New York: Academic Press, 1990.

FLOWERS, N. M. Crise e recuperação demográfica: os Xavánte de Pimentel Barbosa, Mato Grosso. In: SANTOS, R. V.; COIMBRA JR., C. E. (Orgs.). Saúde dos povos indígenas. Rio de Janeiro: Fiocruz, 1994, p. 213-242.

GALVÃO, E. Encontro de sociedades: índios e brancos no Brasil. Rio de Janeiro: Paz e Terra, 1979 (Coleção Estudos Brasileiros, v. 29).

JUNQUEIRA, C. Os índios do Ipavu. Um estudo sobre a vida do grupo Kamaiurá. $2^{\underline{a}}$ ed. São Paulo: Ática, 1978. 
Sexo e desigualdade entre os Kamaiurá e os Cinta Larga. São Paulo: Olho Dágua/Capes, 2002.

IBGE. Tendências demográficas. Uma análise dos resultados da amostra do Censo Demográfico de 2000. Rio de Janeiro: IBGE, 2004 (Coleção Estudos e Pesquisas, 13).

Tendências demográficas:

uma análise dos indígenas com base nos resultados da amostra dos Censos de 1991 e 2000. Rio de Janeiro: IBGE, 2005 (Coleção Estudos e Pesquisas, 16).

MCSWEENEY, K.; ARPS, S. A "demographic turnaround": the rapid growth of indigenous populations in Lowland Latin America. Latin American Research Review, n. 40, p. 3-29, 2005.

MOTTA, J. L. da. A epidemia de sarampo no Xingu. Relatório apresentado à direção do Serviço Nacional de Proteção aos Índios (SPI), 1954.12p. Mimeografado.

MOTTA, L. S. Relatório da epidemia de sarampo no Xingu. Arquivo do Museu do Índio, 1954. Filme 380, fotogramas 254-272. Microfilmado.

ÖBERG, K. Indian tribes of northern Mato Grosso, Brasil. Washington: Smithsonian Institution, Institute of Social Anthropology, 1953 (Publicação no 15).

PAGLIARO, H.; AZEVEDO, M. M.; SANTOS, R. V. Demografia dos povos indígenas no Brasil: um panorama crítico. In: PAGLIARO, H.; AZEVEDO, M. M.; SANTOS, R. V. (Orgs.). Demografia dos povos indígenas no Brasil. Rio de Janeiro: Fiocruz/Abep; 2005, p. 11-32.

PAGLIARO, H. A revolução demográfica dos povos indígenas: a experiência dos Kaiabi do Parque Indígena do Xingu, Mato Grosso. In: PAGLIARO, H.; AZEVEDO, M. M.; SANTOS, R. V. (Orgs.). Demografia dos povos indígenas no Brasil. Rio de Janeiro: Editora Fiocruz/Abep; 2005, p. 79-102.

PAGLIARO, H.; JUNQUEIRA, C. Recuperação demográfica e fecundidade dos Kamaiurá, povo Tupi do Parque Indígena do Xingu, Brasil Central, 1970-2003. Saúde e Sociedade, São Paulo, 16(2):37-47, 2007.
PAGLIARO, H.; CARVALHO, N. S.; RODRIGUES, D.; BARUZZI, R. G. Dinâmica demográfica dos Suyá, povo Jê do Parque Indígena do Xingu, Brasil Central, 19702004. Cadernos de Saúde Pública, Rio de Janeiro, 23(5):1.071-1.081, 2007.

PINTO, N. R. S.; BARUZZI, R. G. Reclusão pubertária masculina em índios do Alto Xingu, Brasil Central. In: BARUZZI, R. G.; JUNQUEIRA, C. (Orgs.). Parque Indígena do Xingu. Saúde, cultura e história. São Paulo: Terra Virgem, 2005, p. 175-188.

RODRIGUES, D. A Unifesp/Escola Paulista de Medicina, o Projeto Xingu e a política de atenção à saúde dos povos indígenas no Brasil. In: BARUZZI, R. G.; JUNQUEIRA, C. (Orgs.). Parque Indígena do Xingu. Saúde, cultura e história. São Paulo: Terra Virgem; 2005, p.259-273.

Relatórios de Atividades do Distrito Especial Indígena do Xingu. São Paulo: Unifesp, 2002. Mimeografado.

SANTOS, R. V.; PAGLIARO, H.; AZEVEDO, M. M. A dinâmica demográfica dos Mucajai Yanomama: uma entrevista com John Early. In: PAGLIARO, H.; AZEVEDO, M. M.; SANTOS, R. V. (Orgs.). Demografia dos povos indígenas no Brasil. Rio de Janeiro: Editora Fiocruz/Abep, 2005, p. 167-192.

SOUZA, L. G. de; SANTOS, R. V. Perfil demográfico da população indígena Xavánte de Sangradouro - Volta Grande, Mato Grosso (1993-1997), Brasil. Cadernos de Saúde Pública, Rio de Janeiro, 17(2):355366, 2001.

STEINEN, K. V. D. Entre os aborígenes do Brasil Central. Separata da Revista do Arquivo, São Paulo, Departamento de Cultura, n. XXXIV e LVIII, tradução de Egon Schaden, 1940, p. 179-206.

O Brasil Central. Rio de Janeiro: Companhia Editora Nacional, 1942.

WONG, L. R.; MORELL, M. G. G.; CARVALHO, R. L. Notas sobre o comportamento reprodutivo dos povos indígenas no período 1991-2000. In: ENCONTRO NACIONAL DE ESTUDOS POPULACIONAIS, XV. Anais... Caxambu: Abep, 2006. Disponível em: $<$ http://www.abep.org.br>. 
WERNER, D. Fertility and pacification among the Mekranoti of Central Brazil.
Human Ecology, v.11, n.2, p. 227-245, 1983.

\section{Resumen}

Dinámica Demográfica de los Kamaiurá, pueblo Tupí del Parque Indígena del Xingu, Mato Grosso, Brasil, 1970-1999

Este trabajo analiza la dinámica demográfica de los Kamaiurá, pueblo del tronco lingüístico Tupí, que, junto con otros nueve pueblos de las lenguas Aruak, Karib, Tupí y Trumai, habitan el Parque Indígena del Xingu (PIX), al norte del Mato Grosso. Las fuentes de datos son las fichas médicas y los libros de registro de eventos vitales del archivo del Programa de Salud de la Universidad Federal de San Pablo en el PIX, alimentado regularmente desde 1965. Las medidas demográficas estimadas son: composición por edad y sexo; tasas brutas de natalidad; tasas de fecundidad total; tasas de mortalidad general y tasas de mortalidad específicas por edad y sexo; mortalidad proporcional por sexo y edad; y tasas de mortalidad infantil. El estudio muestra que los Kamaiurá están experimentando un proceso de recuperación poblacional, con un crecimiento del 3,1\% al año, entre 1970 y 1999. Altos niveles de fecundidad contribuyeron con la expansión de las nuevas generaciones Kamaiurá, garantizando la sobrevivencia física del grupo. Niveles de mortalidad relativamente bajos y estables resultaron de la mejoría de las condiciones de salud, lo que propicia un aumento de la sobrevivencia de los niños, jóvenes, adultos y ancianos, asegurando una mayor longevidad a la población y mantenimiento de su organización social.

Palabras clave: Kamaiurá. Demografía de los pueblos indígenas. Indios de América del Sur. Alto Xingu.

\section{Abstract}

Demographic dynamics of the Kamaiurá, a Tupi people living in the Xingu Indigenous Park, in Mato Grosso, Brazil, 1970-1999

This article analyzes the demographic dynamics of the Kamaiurá, a people of the Tupi linguistic group, which, together with nine other peoples of the Aruak, Karib, Tupi and Trumai languages, inhabit the Xingu Indigenous Parque, in the northern part of the State of Mato Grosso, Brazil. The sources of data are medical records and vital statistics from the health program of the Federal University of São Paulo at Xingu Park, reported regularly since 1965. The demographic measurements included are: composition by age and sex, gross fertility rates, total fecundity rates, general death rate, and specific death rate by age and sex, proportional mortality by age and sex, and child mortality rates. The study shows that the Kamaiurá have been in a process of demographic recovery, showing a growth rate of 3.1\% per year between 1970 and 1999 . High levels of fecundity have contributed to the expansion of new generations of Kamaiurá, guaranteeing the group's physical survival. Relatively low and stable death rates have resulted from improved health conditions, which foster better survival of children, adolescents, adults and the elderly, assuring greater longevity for the population and maintenance of its social organization.

Keywords: Kamaiurá. Demography of Brazilian indigenous peoples. Indians of South America. Upper Xingu. 